\title{
SPIN-WAVE EXCITATIONS AND FLUCTUATIONS OF THE EXCHANGE INTERACTION IN AMORPHOUS $\mathrm{Fe}_{1-x} \mathrm{Zr}_{x}$ ALLOYS
}

\author{
P. KossaCKi ${ }^{a}$, T. Stobiecki ${ }^{b}$ AND H. SzymCzAK ${ }^{a}$ \\ a Institute of Physics, Polish Academy of Sciences \\ Al. Lotników 32/46, 02-668 Warszawa, Poland \\ ${ }^{b}$ Institute of Electronics, Academy of Mining and Metallurgy \\ Mickiewicza 30, 30-059 Kraków, Poland
}

(Received Seplember 18, 1992; revised version February 26, 1993)

Detailed magnetization measurements were performed on amorphous $\mathrm{Fe}_{1-x} \mathrm{Zr}_{x}$ alloys with $0.14 \leq x \leq 0.48$ at temperatures ranging from $4.2 \mathrm{~K}$ to the Curie temperature in external magnetic field up to $5 \mathrm{~T}$. An analysis of the experimental data reveals that the fluctuations of the exchange integral resulting from the structural disorder give important contribution to the thermal demagnetization and high-field susceptibility of Fe-rich alloys. For Zr-rich alloys the effect of fluctuations of the exchange integral is negligible and thermal demagnetization is described in terms of spin-wave excitations. No Stoner single-particle excitations were observed for alloys with $x>0.14$.

PACS numbers: $75.30 . \mathrm{Cr}, 75.50 . \mathrm{Kj}, 75.70 .-\mathrm{i}$

\section{Introduction}

The amorphous $\mathrm{Fe}-\mathrm{Zr}$ alloys have attracted much attention to both experimental and theoretical physicists (see [1] for references). These alloys show unusual compositional dependence of magnetic and transport properties. Depending on the $\mathrm{Fe}$ concentration, the $\mathrm{Fe}_{1-x} \mathrm{Zr}_{\boldsymbol{x}}$ amorphous alloys range from superconductivity for $x>0.62$ to magnetically ordered for $x<0.62$ [2]. Magnetic properties of these alloys for Fe-rich compositions (near $x=0.1$ ) are particularly interesting. They are found to exhibit the Invar-like properties [3] and reentrant spin glass behavior at low temperatures [4]. Furthermore, small-angle neutron scattering measurements [5] have shown that near $x=0.1$ the spin fluctuations are correlated over relatively short ranges of about $5 \mathrm{~nm}$ and the long-range ferromagnetic ordering does not develop at any temperature. These unusual magnetic properties would be a consequence of strong competition between ferromagnetic and antiferromagnetic 
exchange interactions resulting from the fluctuations of the exchange interactions due to structural disorder [6,7].

Effect of the dispersion of the exchange integral on thermal demagnetization and high-field susceptibility of metal-metalloid (Co-based) amorphous alloys have been studied in our previous papers $[8,9]$. It has been shown that the $T^{2}$-type contribution to the thermal demagnetization, identified usually with Stoner single-particle excitations, appears also due to dispersion of the exchange interaction in the amorphous alloys. Fluctuations of the exchange integral have also some effect on the high-field susceptibility. We expect that dispersion of the exchange integral should give contribution to the thermal demagnetization and high-field susceptibility of amorphous metal-metal alloys. To check this assumption we performed detailed measurements of field and temperature dependencies of magnetization in amorphous $\mathrm{Fe}-\mathrm{Zr}$ thin films.

\section{Experimental results}

Amorphous films of $\mathrm{Fe}_{1-x} \mathrm{Zr}_{x}$ (where $0.14<x<0.48$ ) were prepared by the rf-sputtering technique. Before film deposition the system was evacuated to a basic pressure of about $1 \times 10^{-6} \mathrm{mbar}$, and, during sputtering, the argon gas was next introduced to $6 \times 10^{-3}$ mbar pressure. In order to obtain very clean films with the smallest possible amount of impurities of $\mathrm{C}, \mathrm{O}$, and $\mathrm{N}$, we resputtered a composite target consisting of 99.998-pure iron disk $80 \mathrm{~mm}$ in diameter and of quadratic plates of 99.8 -pure $\mathrm{Zr}$ of approximately $5 \mathrm{~mm}^{2}$. The film composition was regulated by changing the number of the plates of iron disk. The atomic concentration of the individual films was measured by energy dispersive X-ray fluorescence analysis with $\pm 1 \%$ accuracy. The composition homogeneity of the films was checked by the Auger electron spectroscopy depth profiling. The sputtering rate was typically $0.13 \mathrm{~nm} / \mathrm{s}$, and samples were made with thicknesses between $850 \mathrm{~nm}$ and $1100 \mathrm{~nm}$. The thicknesses and distributions of thickness were controlled by alpha-step profiling. The samples were deposited on a water-cooled quartz and glass substrate. The amorphous state was checked by electron diffraction, the Mössbauer spectroscopy (e.g. [10]).

It is known that liquid-quenched ribbons of $\mathrm{Fe}-\mathrm{Zr}$ can be obtained as a stable amorphous alloy only in a limited range of concentration, although thin films of this alloy prepared by sputtering are stable in a wider range of concentration (e.g. [11]). Magnetization measurements were performed in magnetic field up to $5 \mathrm{~T}$ in the temperature range from $4.2 \mathrm{~K}$ to $300 \mathrm{~K}$ using a vibrating sample magnetometer (VSM). The results of high-field magnetization measurements for alloys with $x=0.14,0.20,0.35,0.40$, and 0.48 performed at $T=4.2 \mathrm{~K}$ are presented in Figs. 1a-e.

Magnetization curves are analyzed using the following formula:

$$
M(H)=M(0)+\chi_{\mathrm{hr}} H-A H^{-1 / 2}-\Delta M_{\mathrm{sw}}(H, T),
$$

where $\chi_{\mathrm{hr}}$ describes high-field susceptibility. The term $A H^{-1 / 2}$ is concerned with local random anisotropy [12]. This term was predicted also by Chudnowsky [13]. It is assumed that in our alloys local magnetocrystalline anisotropy is rather low 


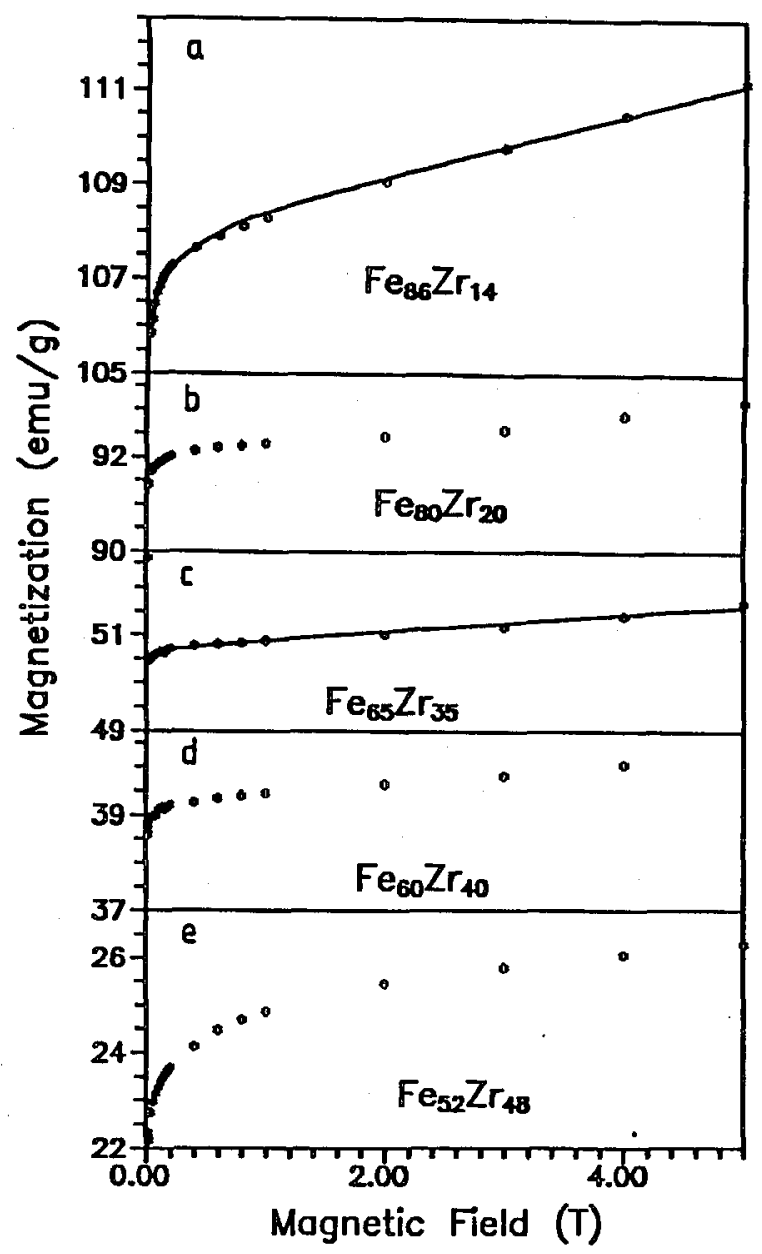

Fig. 1. Magnetization measurements for amorphous $\mathrm{Fe}_{1-x} \mathrm{Zr}_{x}$ alloys as a function of magnetic field for $T=4.2 \mathrm{~K}$. (a) $x=0.14$, (b) $x=0.20$, (c) $x=0.35$, (d) $x=0.40$, (e) $x=0.48$. The solid line is calculated according to Eq. (1) for parameters given in Table.

and therefore random anisotropy model [14] cannot be applied in this case. The last term in Eq. (1) describes the spin-wave excitation and can be estimated from the expression describing the temperature dependence of the magnetization due to spin-wave excitations [8]:

$$
\Delta M_{\mathrm{sw}}(H, T)=g \mu_{\mathrm{B}}\left(\frac{k T}{4 \pi D_{\mathrm{sw}}}\right)^{3 / 2} \sum_{n=1}^{\infty} n^{-3 / 2} \exp \left(-\frac{n g \mu_{\mathrm{B}} H}{k T}\right),
$$

where the symbols have their usual meaning, $D_{\mathrm{sw}}$ being the spin-wave stiffness constant. It should be mentioned that for external field $H>1 \mathrm{~T}$ the spin-wave excitations give negligible contribution to the magnetization curve. 
The set of parameters which gives the best fit to the experimental data is given in Table.

TABLE

Saturation magnetization $M(0)$, high-field susceptibility $\chi_{\text {hf }}$ and parameters $A$ (according to Eq. (1)) and B (Eq. (6)) for amorphous $\mathrm{Fe}_{1-x} \mathrm{Zr}_{x}$.

\begin{tabular}{c|c|c|c|c}
\hline \hline$x$ & $\begin{array}{c}M(0) \\
{\left[\mu_{\mathrm{B}} /(\text { at.Fe })\right]}\end{array}$ & $\begin{array}{c}\chi_{\mathrm{hf}}(4.2) \\
{\left[\mu_{\mathrm{B}} /(\text { at.Fe T) }]\right.}\end{array}$ & $\begin{array}{c}A \\
{\left[\mu_{\mathrm{B}} \mathrm{T}^{1 / 2} /(\text { at.Fe })\right]}\end{array}$ & $\begin{array}{c}B \\
{\left[\mu_{\mathrm{B}} \mathrm{K}^{-3 / 2} /(\text { at.Fe })\right]}\end{array}$ \\
\hline 0.14 & 1.37 & $8.46 \times 10^{-3}$ & $4.63 \times 10^{-3}$ & $1.58 \times 10^{-4}$ \\
0.20 & 1.30 & $3.61 \times 10^{-3}$ & not determined & not determined \\
0.35 & 0.955 & $3.14 \times 10^{-3}$ & $8.03 \times 10^{-3}$ & $2.15 \times 10^{-4}$ \\
0.40 & 0.820 & $4.11 \times 10^{-3}$ & not determined & not determined \\
0.48 & 0.625 & $6.89 \times 10^{-3}$ & not determined & not determined
\end{tabular}

Figures 1a-e and Table show that the saturation magnetization $M(0)$ decreases slowly with decreasing Fe concentration. At the same time high-field susceptibility $\chi_{\mathrm{hf}}$ increases rapidly with decreasing $\mathrm{Zr}$ content. Similar effect is observed on low-Fe concentration side of $\chi_{\mathrm{hr}}(x)$ dependence (see Fig. 2). In both cases the increase in high-field susceptibility is connected with spin-glass behavior expected for amorphous $\mathrm{Fe}_{1-x} \mathrm{Zr}_{\boldsymbol{x}}$ alloys in Fe-rich and $\mathrm{Fe}$-poor regions.

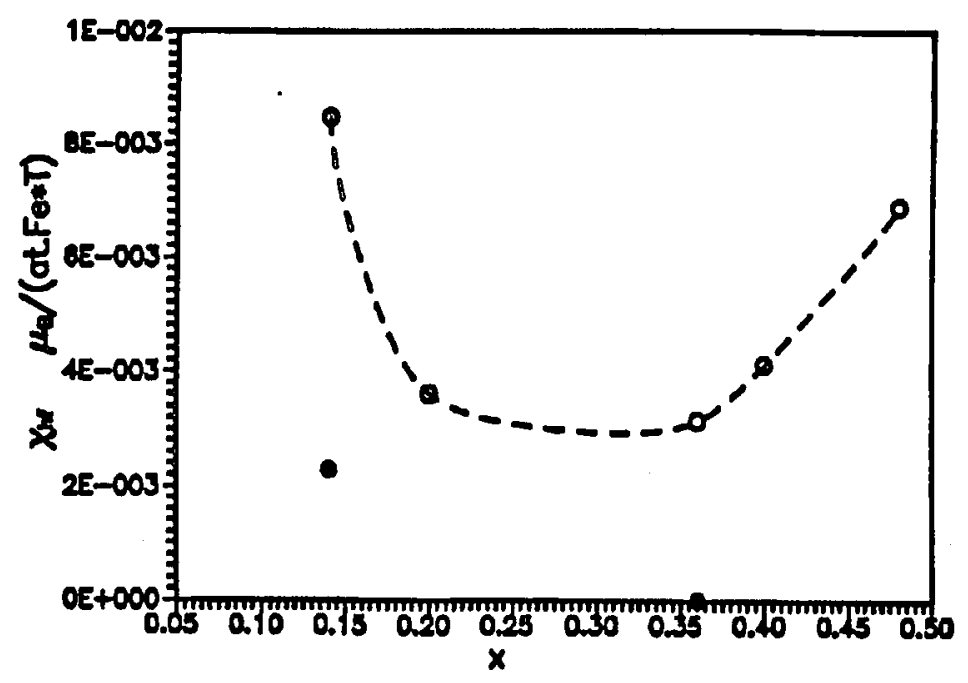

Fig. 2. The concentration dependence of high-field susceptibility $\chi_{\mathrm{h}}$ for amorphous $\mathrm{Fe}_{1-x} \mathrm{Zr}_{x}$ alloys (open circles). For $x=0.14$ and $x=0.35$ calculated values of $\chi_{\mathrm{h} f}$ (according to formula (5)) are marked by full circles. The dashed line is guide to the eye only. 


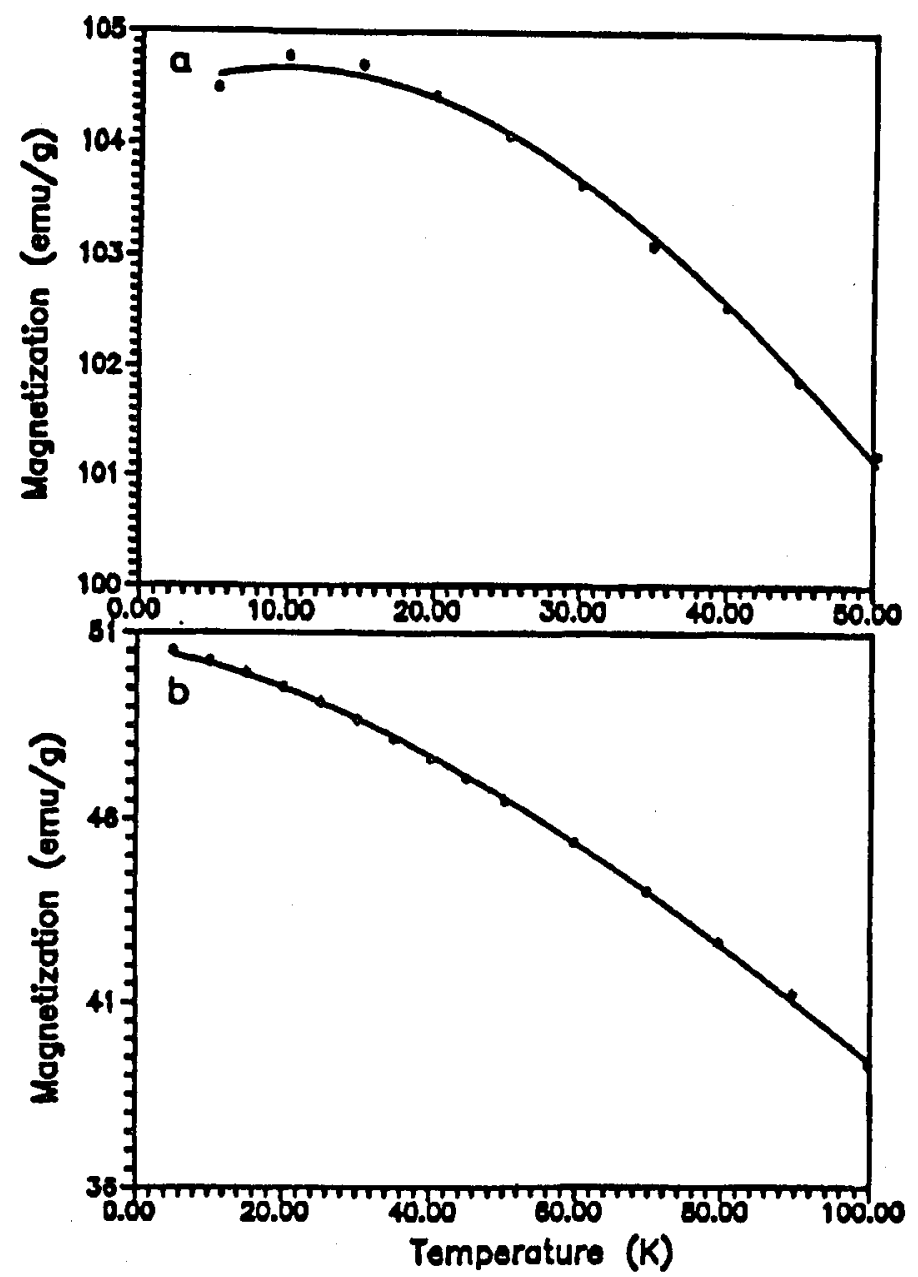

Fig. 3. Temperature dependence of the magnetization $M(0)$ for amorphous $\mathrm{Fe}_{1-x} \mathrm{Zr}_{x}$ alloys. The solid line is calculated according to Eq. (6). (a) $x=0.14$, (b) $x=0.35$.

Figures $3 a$ and $3 b$ present the temperature dependence of magnetization for $x=0.14$ and $x=0.35$ respectively. The fall of magnetization below $10 \mathrm{~K}$ is observed for $x=0.14$. This effect may be compared with rapid increase in $\chi_{\mathrm{hf}}$ observed for $x=0.14$ alloy. Both effects are attributed to a reentrant spin-glass behavior of $\mathrm{Fe}$-rich $\mathrm{Fe}-\mathrm{Zr}$ amorphous alloys [4]. On the other hand, no reentrant behavior is observed for alloys with higher $\mathrm{Zr}$ concentration (see Fig. 3b). Some deviations from linear $M(H)$ dependencies observed in Figs. 1b-d for high values of external magnetic fields $(H>2 \mathrm{~T})$ cannot be explained in terms of existing theories. 


\section{Discussion}

Experimental data are analyzed using the following Hamiltonian [15]:

$$
\mathcal{H}=-\sum_{\langle i j\rangle} J_{i j} \bar{S}_{i} \bar{S}_{j}-g \mu_{\mathrm{B}} \bar{H} \sum_{i} \bar{S}_{i}
$$

where $J_{i j}$ is the exchange integral between $i$-th and $j$-th atom. The first sum is over pairs of the nearest neighbors and the next sum is over individual spins. Exchange integrals are independent and equally distributed according to Gaussian distribution, where $J$ and $\Delta$ are the first and the second moments of $J_{i j}$ distribution. In terms of mean field approximation the following set of equations is obtained:

$$
\begin{aligned}
& \mu=\frac{\left[\left\langle S_{z}\right\rangle\right]_{q}}{S}=(2 \pi)^{-1 / 2} \int_{-\infty}^{\infty} \mathrm{e}^{-t^{2} / 2} B_{S}(X) \mathrm{d} t, \\
& q=\frac{\left[\left\langle S_{z}\right\rangle^{2}\right]_{q}}{S^{2}}=(2 \pi)^{-1 / 2} \int_{-\infty}^{\infty} \mathrm{e}^{-t^{2} / 2} B_{S}^{2}(X) \mathrm{d} t, \\
& X=\left(S^{2} z^{1 / 2} \Delta q^{1 / 2} t+z J S^{2} \mu+g \mu_{\mathrm{B}} H S\right) / k T,
\end{aligned}
$$

where magnetization is $m=N g \mu_{\mathrm{B}} S \mu, q$ represents spin-glass order parameter, $N$ is the number of magnetic ions in $\mathrm{cm}^{3}$ and $z$ is the number of nearest neighbors. At zeroth temperature parameter $q=1$. Thus the susceptibility is given by the formula

$$
\chi=\frac{\left(g \mu_{\mathrm{B}}\right)^{2}}{S z J} \frac{C}{1-C}, \quad C=(2 / \pi)^{1 / 2} \frac{z J}{z^{1 / 2} \Delta} \exp \left[-\frac{z J^{2}}{2 \Delta^{2}}\left(\mu+\frac{\mu_{\mathrm{B}} g H}{S z J}\right)^{2}\right] .
$$

The exchange integral $J$ is determined from the formula describing the Curie temperature: $k T_{\mathrm{C}}=z J S^{2} / 3$. When magnetic field is smaller, then the exchange field $g \mu_{\mathrm{B}} H / S z J \ll \mu$, the magnetization increases linearly with field. Therefore $\Delta M_{\text {dysp }}(H)$ has the form $\Delta M_{\text {dysp }}(H)=\chi_{\mathrm{hf}} H$, where $\chi_{\mathrm{hf}}$ is given by Eq. (5).

Thermal demagnetization was analyzed using the following formula:

$$
M(T)=M(0)-\Delta M_{\text {dysp }}(T)-B T^{3 / 2} .
$$

In Eq. (6) the second term describes thermal demagnetization due to the dispersion of the exchange integral. This term was estimated numerically from Eqs. (4) without any additional fitting parameters. It should be mentioned that for low values of $\Delta$ the leading term in $\Delta M_{\text {disp }}(T)$ is proportional to $T^{2}$ (see Eq. (4) in [8]). The third term in Eq. (6) describes spin-wave contribution to thermal demagnetization (Eq. (2)). From the value of $B$ we determined the spin-wave stiffness constant $D_{\mathrm{sw}}$ (using Eq. (2)) for $x=0.14$ and 0.35 .

The mechanism of thermal demagnetization related to the dispersion of exchange integral is effective only for amorphous $\mathrm{Fe}_{86} \mathrm{Zr}_{14}$ alloy. For this alloy this mechanism is responsible also for the increase in the high-field susceptibility (see Fig. 2). Figures 3a and $3 \mathrm{~b}$ present the comparison of the theoretical values of $M(T)$ (estimated from Eq. (6)) with experimental data. It can be seen that $M(T)$ dependence is adequately described by the theory, at least for a temperature range $4.2 \leq T \leq 0.2 T_{\mathrm{C}}$. It should be mentioned that our experimental data suggest absence of the Stoner single-particle contribution to the demagnetization curves. 


\section{Conclusion}

We have shown that the fluctuations of the exchange integral give important contribution to thermal demagnetization and high-field susceptibility of amorphous $\mathrm{Fe}_{86} \mathrm{Zr}_{14}$ alloys. For $x \geq 0.14$ thermal demagnetization of amorphous $\mathrm{Fe}_{1-x} \mathrm{Zr}_{x}$ alloys is determined only by spin-wave excitations. We did not observe any effects related to the Stoner single-particle excitations.

This work was in part supported by the Committee for Scientific Research grant no. 204659101.

\section{References}

[1] S.N. Kaul, V. Siruguri, G. Chandra, Phys. Rev. B 45, 12343 (1992).

[2] Z. Altounian, J.O. Ström-Olsen, Phys. Rev. B 27, 4149 (1982).

[3] K. Shirakawa, S. Ohnuma, M. Nose, T. Masumoto, IEEE Trans. Magn. 16, 910 (1980).

[4] H. Hiroyoshi, K. Fukamichi, J. Appl. Phys. 53, 2226 (1982).

[5] J.J. Rhynne, G.E. Fish, J. Appl. Phys. 57, 3407 (1985).

[6] R.A. Cowley, C. Patterson, N. Cowlam, P.K. Ivison, J. Martinez, L.D. Cussen, J. Phys. Condens. Matter 3, 9251 (1991).

[7] T. Kaneyoshi, J. Phys. C 8, 3415 (1975).

[8] F.R. de Boer, J.C.P. Klaasse, P. Kossacki, H. Nakotte, P. Puźniak, A. Szewczyk, H. Szymczak, X.P. Zhong, J. Magn. Magn. Mater. 104-107, 113 (1992).

[9] F.R. de Boer, P. Kossacki, P. Puźniak, T. Stobiecki, H. Szymczak, X.P. Zhong, J. Magn. Magn. Mater. 101, 3 (1991).

[10] M. Przybylski, K. Krop, T. Stobiecki, L. Dargel-Sulir, Hyperfine Interact. 27, 425 (1986).

[11] N. Saito, K. Fukamichi, Y. Nakagawa, Sci. Rep. Res. Inst. Tohoku Univ. A 35, 65 (1990).

[12] M. Vazquez, W. Fernengel, H. Kronmüller, Phys. Status Solidi A 115, 547 (1989).

[13] E.M. Chudnowsky, J. Appl. Phys. 64, 5770 (1988).

[14] B. Barbara, J. Filippi, A. Marchand, P. Mollard, V.S. Amaral, X. Devaux, A. Rousset, J. Phys. I (France) 2, 101 (1992).

[15] P. Kossacki, J. Magn. Magn. Mater., accepted for publication. 